\title{
AXION IN A 3-3-1 MODEL WITH RIGHT-HANDED NEUTRINOS
}

\author{
DANG VAN SOA \\ Department of Physics, Hanoi University of Education, Hanoi, Vietnam \\ TRAN DINH THAM \\ Pham Van Dong University, Quang Ngai Viet Nam \\ Email:dvsoa@hnue.edu.vn; tdtham@pdu.edu.vn \\ Received 31 August 2013; \\ Accepted for publication 18 September 2013
}

\begin{abstract}
We show that Peccei - Quinn $(P Q)$ symmetry can be a natural outcome in the 3-31 model with right-handed neutrinos after imposing a $Z_{11} \otimes Z_{2}$ symmetry. This symmetry is suitable accommodated in this model when we augmented its spectrum by including a singlet scalar field with large $V E V$. We work out the breaking of the $P Q$ symmetry, yielding the axion, and study the decay of axion into two photons. The result shows that the lifetime of axion is very long, therefore the axion can play the role of the late decaying particle(LDP) in a dark matter and it is stable in our universe.
\end{abstract}

\section{INTRODUCTION}

The most attractive candidate for solution of the strong-CP problem is PQ mechanism, where the $\mathrm{CP}$-violating phase $\theta$ is explained by the existence of a new pseudoscalar field, called the axion [1]. At present, the axion mass is constrained by laboratory searches [2] and by astrophysical and cosmological considerations [3] to between $10^{-6} \mathrm{eV}$ and $10^{-3} \mathrm{eV}$. If the axion has a mass near the low limit of order $10^{-5} \mathrm{eV}$, it is a good candidate for the dark matter of the universe [4]. Besides that, an axino (the fermionic partner of the axion ) naturally appears in SUSY models [5,6], which acquires a mass from three - loop Feynman diagrams in a typical range between a few $\mathrm{eV}$ up to a maximum of 1 $\mathrm{keV}[7,8]$. Recently, the candidates for dark matter have been done in extended models of the standard model, such as in the 3-3-1 models [9,10], or in extra-dimensional gauge fields [11].

In Refs. [9, 10], authors have studied the consequences of discrete symmetries in versions of the 3-3-1 model. The important point is that, a PQ symmetry is almost automatic in the classical Lagrangian of 3-3-1 models. It is only necessary to avoid a trilinear term in the scalar potential by introducing a $Z_{2}$ symmetry. Unfortunately even in this case the PQ symmetry is explicitly broken by gravity effects. In order to stabilize the axion, we must introduce local discrete symmetry $Z_{N}$. In this paper we show that PQ symmetry can be a natural outcome in the 3-3-1 model with right-handed neutrinos after imposing a $Z_{11} \otimes Z_{2}$ symmetry. After breaking the PQ symmetry yielding the axion we study the 
decay of axion into two photons in detail. Our purpose is to show that axion in the 3-3-1 model with right-handed neutrinos can play the role of LDP in the dark matter and it is stable in our universe. The paper is organized as follows. In Sec. II we briefly present the general structure of the 3-3-1 model with right-handed neutrinos. In Sec. III we carry out the analysis of PQ symmetry and axion. In Sec. IV we study the decay of axion into two photons in detail. We outline our main results in the last section (Sec. V).

\section{A REVIEW OF THE MODEL}

The particle content in this model, which is anomaly free, is given as follows

$$
\psi_{a L}=\left(\nu_{a L}, l_{a L}, N_{a L}\right)^{T} \sim(1,3,-1 / 3), \quad l_{a R} \sim(1,1,-1),
$$

where $a=1,2,3$ is a family index. Here the right-handed neutrino is denoted by $N_{L} \equiv$ $\left(\nu_{R}\right)^{c}$.

$$
\begin{aligned}
Q_{i L} & =\left(d_{i},-u_{i}, d_{i L}^{\prime}\right)_{L}^{T} \sim(3, \overline{3}, 0), \quad Q_{3 L}=\left(u_{3}, d_{3}, u_{3}^{\prime}\right)_{L}^{T} \sim(3,3,1 / 3), \quad i=1,2, \\
u_{i R} & \sim(3,1,2 / 3), \quad d_{i R} \sim(3,1,-1 / 3), \quad U_{3 R} \sim(3,1,2 / 3), \quad d_{3 R} \sim(3,1,-1 / 3)(2)
\end{aligned}
$$

Electric charges of the exotic quarks $U$ and $D_{\alpha}$ are the same as of the usual quarks, i.e. $q_{U}=\frac{2}{3}$ and $q_{D_{\alpha}}=-\frac{1}{3}$. In order to generate the masses for the gauge bosons and fermions, the model requires three triplets of scalars, namely

$$
\begin{gathered}
\chi^{T}=\left(\chi^{0}, \chi^{-}, \chi^{\prime}\right) \sim\left(1,3,-\frac{1}{3}\right), \\
\rho^{T}=\left(\rho^{+}, \rho^{0}, \rho^{\prime}\right) \sim(1,3,2 / 3), \\
\eta^{T}=\left(\eta^{0}, \eta^{-}, \eta^{\prime 0}\right) \sim(1,3,-1 / 3) .
\end{gathered}
$$

The Yukawa interactions which induce masses for the fermions can be written in the most general form as

$$
\begin{aligned}
\left(\mathcal{L}_{Y}^{\chi}+\mathcal{L}_{Y}^{\phi}\right)= & G_{1} \bar{Q}_{i L} \chi u_{3 R}^{\prime}+G_{2}^{i j} \bar{Q}_{i L} \chi^{*} d_{j R}^{\prime}+G_{3}^{a} \bar{Q}_{3 L} \eta u_{a R} \\
& +G_{4}^{i a} \bar{Q}_{i L} \eta^{*} d_{a R}+G_{5}^{3 a} \bar{Q}_{3 L} \rho d_{a R} \\
& +G_{6}^{i a} \bar{Q}_{i L} \rho * u_{a R}+h_{a b} \bar{f}_{a L} \rho e_{b R} \\
& +h_{a b}^{\prime} \epsilon^{i j k}\left(\bar{f}_{a L}\right)_{i}\left(f_{b L}\right)_{j}^{c}\left(\rho^{*}\right)_{k}+\text { h.c. }
\end{aligned}
$$

where repeated indices mean summation.

In order to complete the spectrum we include an additional singlet scalar field, $\phi \sim(1,1,0)$, allowing for the desired discrete symmetry which will enable us to get an axion protected under large gravitational contribution to its mass. We can write the most 
general potential for this model, renormalizable and gauge invariant

$$
\begin{aligned}
V_{H}= & \mu_{\phi}^{2} \phi^{2}+\mu_{\chi}^{2} \chi^{2}+\mu_{\rho}^{2} \rho^{2}+\mu_{\eta}^{2} \eta^{2} \\
& +\lambda_{1} \chi^{4}+\lambda_{2} \eta^{4}+\lambda_{3} \rho^{4}+\lambda_{4}\left(\chi^{+} \chi\right)\left(\eta^{+} \eta\right)+\lambda_{5}\left(\chi^{+} \chi\right)\left(\rho^{+} \rho\right) \\
& +\lambda_{6}\left(\eta^{+} \eta\right)\left(\rho^{+} \rho\right)+\lambda_{7}\left(\chi^{+} \eta\right)\left(\eta^{+} \chi\right)+\lambda_{8}\left(\chi^{+} \rho\right)\left(\rho^{+} \chi\right)+\lambda_{9}\left(\eta^{+} \rho\right)\left(\rho^{+} \eta\right) \\
& +\lambda_{10}\left(\phi \phi^{*}\right)^{2}+\lambda_{11}\left(\phi \phi^{*}\right)\left(\chi^{+} \chi\right)+\lambda_{12}\left(\phi \phi^{*}\right)\left(\rho^{+} \rho\right)+\lambda_{13}\left(\phi \phi^{*}\right)\left(\eta^{+} \eta\right)
\end{aligned}
$$

and

$$
\begin{aligned}
& V_{N H}=\mu_{\chi \eta}^{2} \chi^{+} \eta+f_{1} \chi^{+} \eta \phi+f_{2} \chi^{+} \eta \phi^{*}+\lambda_{14}\left(\chi^{+} \eta\right)^{2}+\lambda_{15} \chi^{+} \eta \phi \phi+\lambda_{16} \chi^{+} \eta \phi^{*} \phi+\lambda_{17} \chi^{+} \eta \phi^{*} \phi^{*} \\
& \quad+\frac{1}{\sqrt{2}} \epsilon^{i j k}\left(f_{3} \eta_{i} \rho_{j} \chi_{k}+f_{4} \eta_{i} \eta_{j} \rho_{k}+f_{5} \chi_{i} \chi_{j} \rho_{k}\right)+\epsilon^{i j k}\left(\lambda_{18} \eta_{i} \rho_{j} \chi_{k}+\lambda_{19} \eta_{i} \eta_{j} \rho_{k}+\lambda_{20} \chi_{i} \chi_{j} \rho_{k}\right) \phi \\
& \quad+\epsilon^{i j k}\left(\lambda_{21} \eta_{i} \rho_{j} \chi_{k}+\lambda_{22} \eta_{i} \eta_{j} \rho_{k}+\lambda_{23} \chi_{i} \chi_{j} \rho_{k}\right) \phi^{*}+\lambda_{24}\left(\chi^{+} \rho\right)\left(\rho^{+} \eta\right)+\lambda_{25}\left(\chi^{+} \eta\right)\left(\eta^{+} \eta\right) \\
& \quad+\lambda_{26}\left(\chi^{+} \eta\right)\left(\rho^{+} \rho\right)+\lambda_{27}\left(\chi^{+} \eta\right)\left(\chi^{+} \chi\right)+H . c,
\end{aligned}
$$

where $V_{H}$ and $V_{N H}$ are the hermintian and non-hermintian terms of the potential, respectively. Here we have all the necessary ingredients concerning the discrete symmetry to the model.

\section{PQ SYMMETRY AND AXION}

A discrete symmetry $Z_{N}$ can naturally be accommodated when the theory has enough number of fields in spectrum [9]. In order to seek for the valid solution to the strong -CP problem, let us proceed further by first assigning the correct $Z_{11}$ charges to the fields. Defining $\omega \equiv e^{2 \pi i \frac{k}{11}},\{k=0, \pm 1, \ldots \pm 5\}$ the $Z_{11}$ transformations are given by

$$
\begin{aligned}
\phi & \rightarrow \omega_{1} \phi, f_{a L} \rightarrow \omega_{1}^{-1} f_{a L}, \rho \rightarrow \omega_{1}^{-1} \rho, d_{a R} \rightarrow \omega_{2}^{-1} d_{a R}, \\
\chi & \rightarrow \omega_{3} \chi,\left(e_{R}, u_{3 R}^{\prime}\right) \rightarrow \omega_{3}^{-1}\left(e_{R}, u_{3 R}^{\prime}\right), Q_{i L} \rightarrow \omega_{4} Q_{i L}, \\
d_{i R}^{\prime} \rightarrow \omega_{4}^{-1} d_{i R}^{\prime}, \eta \rightarrow \omega_{5} \eta, u_{a R} \rightarrow \omega_{5}^{-1} u_{a R}, Q_{3 L} & \rightarrow \omega_{0} Q_{3 L} .
\end{aligned}
$$

Beside the $Z_{11}$ symmetry we impose a $Z_{2}$ symmetry that acts as

$$
\left(\phi, \chi, d_{R}^{\prime}, u_{3 R}^{\prime}\right) \rightarrow-\left(\phi, \chi, d_{R}^{\prime}, u_{3 R}^{\prime}\right)
$$

With the remaining fields transforming trivial, the only term which remains in the nonhermitean potential is $\eta \rho \chi \phi$. We have the stage settled to see that an automatic PQ arise in the model. We start by assigning the PQ quantum numbers such that quarks of opposite chiralities have opposite charges, yielding chiral quarks under $U_{P Q}$ transformation as

$$
\begin{gathered}
u_{a L} \rightarrow e^{-i \alpha X_{u}} u_{a L}, u_{a R} \rightarrow e^{i \alpha X_{u}} u_{a R}, u_{3 L}^{\prime} \rightarrow e^{-i \alpha X_{u}^{\prime}} u_{3 L}^{\prime}, u_{3 R}^{\prime} \rightarrow e^{i \alpha X_{u}^{\prime}} u_{3 R}^{\prime}, \\
d^{a L} \rightarrow e^{-i \alpha X_{d}} d_{a L}, d^{a R} \rightarrow e^{i \alpha X_{d}} d_{a R}, d_{i L}^{\prime} \rightarrow e^{-i \alpha X_{d}^{\prime}} d_{i L}^{\prime}, d_{i R}^{\prime} \rightarrow e^{i \alpha X_{d}^{\prime}} d_{i R}^{\prime} .
\end{gathered}
$$

For the leptons we can define their PQ charges by

$$
e_{a L} \rightarrow e^{i \alpha X_{e}} e_{a L}, e_{a R} \rightarrow e^{i \alpha X_{e}} e_{a R}, \nu_{a L} \rightarrow e^{i \alpha X_{\nu}} \nu_{a L}, \nu_{a R} \rightarrow e^{i \alpha X_{\nu R}} \nu_{a R}
$$


From The Yukawa couplings as well as the non-hermitean term $\eta \rho \chi \phi$, we easily see that PQ charges for the scalars are constrained and imply the following relations

$$
X_{d}=-X_{u}, X_{d^{\prime}}=X_{u^{\prime}}, X_{\nu}=X_{e R}, X_{e}=X_{\nu R}
$$

We can make the further choice $X_{d}=X_{d^{\prime}}$, leading to

$$
X_{d}=X_{d^{\prime}}=-X_{u}=-X_{u^{\prime}}=-X_{e}=X_{e R}=X_{\nu}=-X_{\nu R}
$$

implying that the PQ symmetry is chiral for the lepton too and the scalars transform as

$$
\begin{array}{r}
\phi \rightarrow e^{-2 i \alpha X_{d}} \phi, \eta^{0} \rightarrow e^{2 i \alpha X_{d}} \eta^{0}, \eta^{-} \rightarrow \eta^{-}, \eta^{\prime 0} \rightarrow e^{2 i \alpha X_{d}} \eta^{\prime 0}, \\
\rho^{+} \rightarrow \rho^{+}, \rho^{0} \rightarrow e^{-2 i X_{d}} \rho^{0}, \rho^{\prime+} \rightarrow \rho^{\prime+}, \chi^{0} \rightarrow e^{-2 i \alpha X_{d}} \chi^{0}, \\
\chi^{-} \rightarrow \chi^{-}, \chi^{\prime 0} \rightarrow e^{2 i \alpha X_{d}} \chi^{\prime 0} .
\end{array}
$$

We note here that the condition $X_{d}=-X_{u}$ is allowed in the context of the standard model since in this case it is not possible to shift the $\bar{\theta}$. Finally, the most general potential invariant under the 3-3-1 model and PQ symmetry ( or $Z_{11} \otimes Z_{2}$ ) is written as

$$
V(\eta, \rho, \chi)=V_{H}+\lambda_{\phi} \epsilon^{i j k} \eta_{i} \rho_{j} \chi_{k} \phi+H . c .
$$

This potential allows us to obtain the mass eigenstate for the scalars, so we can identify the Goldstones which are absorbed by the massive gauge bosons an extract the axion in terms of the interaction eigenstates. To accomplish this, let us consider that only $\chi^{\prime 0}, \rho^{0}, \eta^{0}$ and $\phi$ develop a vacuum expectation value (VEV)and expand such fields around their VEV's in the standard way

$$
\begin{array}{r}
\eta^{0}=\frac{1}{\sqrt{2}}\left(v_{\eta}+R_{\eta}+i I_{\eta}\right), \chi^{\prime 0}=\frac{1}{\sqrt{2}}\left(v_{\chi^{\prime}}+R_{\chi^{\prime}}+i I_{\chi^{\prime}}\right), \\
\phi=\frac{1}{\sqrt{2}}\left(v_{\phi}+R_{\phi}+i I_{\phi}\right), \rho^{0}=\frac{1}{\sqrt{2}}\left(v_{\rho}+R_{\rho}+i I_{\rho}\right) .
\end{array}
$$

Regarding the pseudo-scalars, we can easily obtain the Goldstone bosons and identify the axion $[10]$

$$
a \simeq \frac{1}{\sqrt{1+V^{2}}}\left(I_{\phi}-V I_{\chi^{\prime}}\right)
$$

where $V=\frac{v_{\chi^{\prime}}}{v_{\phi}}$.

Let us remark that, if $v_{\phi} \gg v_{\chi^{\prime}}$ then axion of the model is imaginary part of the singlet $\phi$, as expected.

\section{AXION DECAY INTO TWO PHOTONS}

From Eq.(18) we can see that axion in this model has small component of $I_{\chi^{\prime}}$, so axion is invisible being almost exclusively the imaginary part of the singlet $\phi$, which couples only to the exotic quarks. This to conclude that axion in this model can be the candidate for cold dark mater. For more detail, now we calculate the decay of axion into 
two photons in detail. The coupling of axion with photon is defined through the effective Lagrangian term

$$
L_{a \gamma \gamma}=\frac{\bar{c}_{a \gamma \gamma}}{32 \pi^{2} v_{P Q}} a(x) \widetilde{F}_{\mu \nu} F^{\mu \nu}
$$

In this model only exotic quarks participate in the loop leading to the above anomaly term, which leads to [10]

$$
\bar{c}_{a \gamma \gamma}=-\frac{2}{3 v_{P Q}} \sum X_{q^{\prime}} Q_{q^{\prime}}^{2} \simeq 0.44
$$

If the axion is light enough in which it's mass is comparable to gravitino mass, then the axion can not decay into two gluons but decays into two photons. The amplitude for the decay of axion into two photons is

$$
<f|M| i>=\frac{i \bar{c}_{a \gamma \gamma}}{32 \pi^{2} v_{P Q}} \epsilon_{\lambda}\left(k_{1}\right) k_{1 \alpha} k_{2 \mu} \epsilon^{\alpha \lambda \sigma \mu} \epsilon_{\sigma}\left(k_{2}\right)
$$

After some calculations, we obtain the decay rate

$$
\Gamma(a \rightarrow \gamma \gamma)=\frac{1}{64 \pi}\left(\frac{\bar{c}_{a \gamma \gamma}}{32 \pi^{2} v_{P Q}}\right)^{2} m_{a}^{3}
$$

Note that the value $\Gamma$ varies very widely because of the strong dependence on the axion mass.

For the axion mass in region $10^{-6} \mathrm{eV} \leq m_{a} \leq 10^{-3} \mathrm{eV}$ and note that $[\mathrm{GeV}]^{1}=\frac{1}{6.6 \times 10^{-25}} \mathrm{sec}^{-1}[12]$, the dependence of the decay rate and the lifetime of the axion on its mass is shown in Table 1

\section{Table 1}

\begin{tabular}{|c|c|c|c|c|}
\hline$m_{a}(\mathrm{eV})$ & $10^{-3}$ & $10^{-4}$ & $10^{-5}$ & $10^{-6}$ \\
\hline$\Gamma\left(\mathrm{sec}^{-1}\right) \times 9.4$ & $10^{-64}$ & $10^{-67}$ & $10^{-70}$ & $10^{-73}$ \\
\hline$\tau(\mathrm{sec}) \times 1.06$ & $10^{63}$ & $10^{66}$ & $10^{69}$ & $10^{72}$ \\
\hline
\end{tabular}

From the Table 1, we can see that the lifetime of axion is very long, therefore the axion can be a good candidate for LDP in the dark mater. It is interesting to note that axion lifetime is much larger the age of the universe $\left(\sim 10^{17} \mathrm{sec}\right)$, therefore axion oscillation still exists until now.

\section{CONCLUSIONS}

In this paper we have presented the 3-3-1 model with right-handed neutrinos and show that PQ symmetry can be a natural outcome in this model after imposing the $Z_{11} \otimes Z_{2}$ symmetry. We work out the breaking of the PQ symmetry, yielding the light axion, and study the decay of axion into two photons in detail. The result shows that the lifetime of axion is very long, therefore the axion can play the role of the late decaying massive particle in the dark matter and it is stable in our universe. 


\section{ACKNOWLEDGEMENTS}

The work is supported in part by the National Foundation for Science and Technology Development (NAFOSTED) of Vietnam under grant No. 103.03 - 2012.80.

\section{REFERENCES}

[1] R. D. Peccei and H. Quinn, Phys. Rev. Lett. 1440 (1977) 38; Phys. Rev. D 16 (1977) 1792

[2] J. E. Kim, Phys. Rep. 150, 1 (1987); H. Y. Cheng, ibid. 158, 1 (1988); R. D. Peccei, in CP Violation, edited by C. Jarlskog, Advanced Series on Directions in High Energy Physics, Vol. 3, World Scientific, Singapore, 1989.

[3] M. S. Turner, Phys. Rep. 197 (1990) 67; G. G. Raffelt, ibid. 198 (1990) 1; E. W. Kolb and M. S. Turner, The Early Universe, Addison- Wesley Publ. Company, 1990.

[4] S. Perlmutter et al., Astrophys. J. 517, (1999) 565

[5] J. E. Kim, Phys. Lett. B 136 (1984) 387; J. E. Kim and H. P. Nilles, ibid. 138 (1984) 150.

[6] K. Rajagopal, M. S. Turner, and F. Wilczek, Nucl. Phys. B 358 (1991) 447

[7] M.I. Vysotsky and M. B. Voloshin, Yad. Fiz. 44 (1986) 845; M. B. Voloshin, M.I. Vysotsky and L. B. Okun, Zh. Eksp. Teor. Fiz. 91 (1986) 745 [Sov. Phys. JETP 64 (1986) 446].

[8] J. E. Kim, A. Masiero, and D. V. Nanopoulos, Phys. Lett. B 139, 346 (1984).

[9] Alex G.Dias et al., Phys. Rev. D 68 (2003) 115009

[10] Alex G.Dias, V.Pleitez, Phys. Rev. D 69 (2004) 077702

[11] Y. Burnier and F. Kuhnel, Phys. Rev. D 83 (2011) 115002.

[12] L. N. Thuc and D. V. Soa, Acta Physica 56(4) (2006) 485 